\title{
Avaliação da Morfologia e Propriedades Termo-Mecânicas em Nanocompósitos de PVC
}

\author{
Rodrigo P. da Silva \\ Programa de Pós Graduação em Ciência de Materiais, UFRGS
}

\author{
Raquel S. Mauler, Ricardo V. B. de Oliveira \\ Instituto de Química, UFRGS
}

\author{
Cleia A. Salles \\ Instituto Federal Sul-Rio-Grandense, IFSul
}

\begin{abstract}
Resumo: A busca pela utilização de cargas de reforço aos materiais poliméricos tem sido o grande alvo dos pesquisadores e da indústria. Neste trabalho nanocompósitos de PVC foram obtidos mediante a mistura no estado fundido utilizando-se uma extrusora monorosca. Adicionalmente, três diferentes concentrações de montmorilonita organofílica 1, 2 e 5\% em massa foram incorporadas. Estes foram caracterizados por suas morfologias, propriedades térmicas e termo-dinâmico-mecânicas. O grau de intercalação e esfoliação dos nanocompósitos foi caracterizado por MET. As análises termogravimétricas não demonstraram um aumento na estabilidade térmica dos nanocompósitos com a concentração de argila.
\end{abstract}

Palavras-chave: Nanocompósitos, montmorilonita organofilica, morfologia, PVC.

\section{Evaluation of Mechanical-Thermal Properties and Morphological in PVC Nanocomposites}

Abstract: The search for the use of reinforcement fillers into polymeric materials has been the great objective of researchers and industry. In this work PVC nanocomposites were obtained by melt blending in a single-screw extruder. Additionally, three different organophilic montmorillonite concentrations of 1,2 and 5\% wt were incorporated. These were characterized by morphology, thermal and thermo-dynamic-mechanical properties. The degree of intercalation and exfoliation of nanocomposites was characterized by MET. The thermogravimetric analysis did not show an increase in the thermal stability of the nanocomposites with the clay content.

Keywords: Nanocomposites, organophilic montmorillonite, morphology, PVC.

\section{Introdução}

Nos dias de hoje a incorporação das nanocargas em materiais poliméricos está sendo uma prática muito visada tanto pelos pesquisadores quanto pelas indústrias, com interesse principal de melhorar as propriedades destes polímeros para aumentar significativamente sua gama de aplicações. Algumas destas propriedades são: mecânicas, térmicas, ópticas e magnéticas ${ }^{[1]}$. Pode-se dizer que a principal vantagem destes materiais namométricos é que não há a necessidade de grandes concentrações de cargas, conseguindo-se melhoria de propriedades com níveis de incorporação inferior aos compósitos convencionais.

Um dos polímeros mais utilizados nas indústrias e por pesquisadores para a incorporação das nanocargas é o poli (cloreto de viníla) (PVC). Este polímero é um dos mais versáteis dentre os termoplásticos ${ }^{[2]}$. Devido à necessidade do composto de PVC ser formulado mediante a incorporação de aditivos, este pode ter suas características alteradas dentro de um amplo espectro de propriedades de acordo com sua aplicação final. Assim, pode variar desde o rígido ao extremamente flexível, passando por aplicações que vão desde tubos e perfis rígidos para uso na construção civil, até brinquedos e laminados flexíveis para acondicionamento de sangue e plasma. A grande versatilidade do PVC devese, em parte, também à sua adequação aos mais variados processos de moldagem, podendo ser moldado por injeção, extrusão, calandragem, espalmagem, entre outros ${ }^{[3,4]}$.

Nos nanocompósitos poliméricos, as argilas do grupo das esmectitas, como a montmorilonita, são de longe as mais estudadas, podendo ser encontradas com facilidade (fonte natural), possuindo baixo custo comparado com outras nanocargas ${ }^{[5,6]}$. A MMT tem uma estrutura tipo camadas 2:1, uma camada sanduíche de alumina octaédrica entre duas camadas de sílica tetraédrica. A unidade básica das camadas

Autor para correspondência: Ricardo V. B. de Oliveira, Instituto de Química, Departamento de Química Orgânica, UFRGS, Av. Bento Gonçalves, 9500, Agronomia, CEP: 91501-970, Porto Alegre, RS, Brasil. E-mail: ricardo.oliveira@iq.ufrgs.br 
tetraédricas externas é o óxido de silício, onde os átomos de silício estão ligados a quatro átomos de oxigênio ${ }^{[7-10]}$.

Este trabalho teve como objetivo avaliar o efeito das diferentes concentrações da montmorilonita organicamente modificada em um composto de PVC flexível na dispersão, distribuição e seu reflexo nas propriedades finais dos nanocompósitos preparados.

\section{Experimental}

\section{Materiais}

Neste trabalho, utilizou-se como matriz polimérica para o nanocompósito o composto de poli (cloreto de viníla) (PVC) flexível, valor K $65 \pm 1$, sob código NORVIC SP 1000 produzido pela Braskem S/A. Os aditivos utilizados na formulação do composto de PVC flexível foram: plastificante DOP, estabilizante, lubrificante interno e externo. Entretanto, a exata composição está sob sigilo industrial. O plastificante utilizado foi ftalato de dioctila (DOP) da Elekeiroz S/A, massa molar média de $390 \mathrm{~g} \cdot \mathrm{mol}^{-1}$, densidade de $0,98 \mathrm{~g} . \mathrm{cm}^{-3}$. O DOP é o plastificante mais comumente utilizado na preparação de composto de PVC flexível. A nanoargila mineral utilizada foi a montmorilonita organofílica (OMMT) do grupo das esmectitas, modificada organicamente com um sal de alquil quaternário de amônio, o qual possui dois grupos hidróxietila, uma metila e uma cauda alquílica (T) com comprimento principal de 18 carbonos, ligados ao íon amônio. A OMMT possui densidade de $1,98 \mathrm{~g} \cdot \mathrm{cm}^{-3}$ e grau de modificação de 0,9 meq. $\mathrm{g}^{-1}$, sendo fornecida pela Southern Clay Products, Inc., sob o código Cloisite ${ }^{\circledR}$ 30B.

\section{Obtenção dos nanocompósitos}

Foi misturado o composto de PVC com a argila em um misturador da $\mathrm{MH}$ Equipamentos Ltda, com velocidade de rotação de $1710 \mathrm{rpm}$ por 10 minutos, a uma temperatura de aproximadamente $40{ }^{\circ} \mathrm{C}$ gerada somente pelo efeito cisalhante dos materiais com as hélices do equipamento. Foram variadas as concentrações em massa de OMMT de 1, 2 e $5 \%$ e designado por PVC/OMMT1 para $1 \%$ em massa, PVC/OMMT2 para 2\% em massa e PVC/OMMT5 para 5\% em massa. Posteriormente as misturas foram processadas em uma extrusora granuladora monorosca da Beplast Ltda, velocidade de rotação de $140 \mathrm{rpm}$ e temperaturas de 90 a $100{ }^{\circ} \mathrm{C}$ (direção funil-matriz).

\section{Caracterização}

$\mathrm{Na}$ analise de microscopia eletrônica de transmissão (MET), as amostras foram inicialmente embutidas em resina epóxi por serem extremamente flexíveis. O equipamento utilizado foi um Jeol JEM 1200 EXII.

Os experimentos de análise termo-dinâmico-mecânica (DMTA) foram realizados em um equipamento T800 da TA Instruments, em uma faixa de temperatura de -80 a $120{ }^{\circ} \mathrm{C}$, taxa de aquecimento de $3{ }^{\circ} \mathrm{C} /$ min e freqüência fixa de $1 \mathrm{~Hz}$.
O equipamento utilizado para análise termogravimétrica (TGA) foi um Q50 da TA Instruments, com uma taxa de aquecimento de $20^{\circ} \mathrm{C} / \mathrm{mim}$ sob atmosfera de nitrogênio em uma rampa de aquecimento de 20 até $800{ }^{\circ} \mathrm{C}$.

\section{Resultados e Discussão}

As micrografias de MET mostram as morfologias dos nanocompósitos de PVC/OMMT e estão demonstradas na Figura 1.

Como pode ser visualizada, a dispersão da argila na matriz do PVC tende a formar alguns aglomerados com o aumento da concentração de $1 \%$ para $5 \%$, por outro lado, parece apresentar um maior grau de intercalação e esfoliação

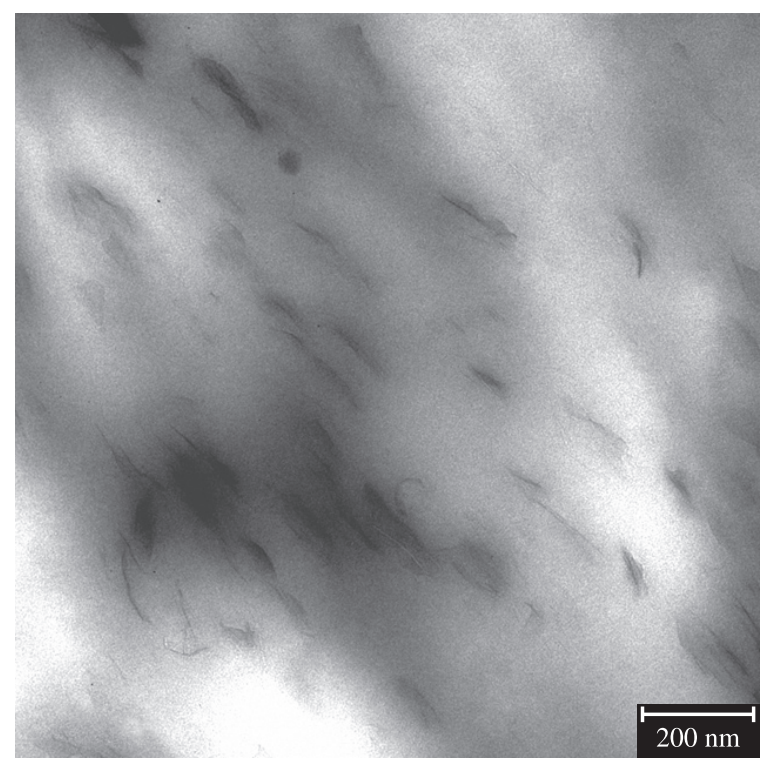

(a)

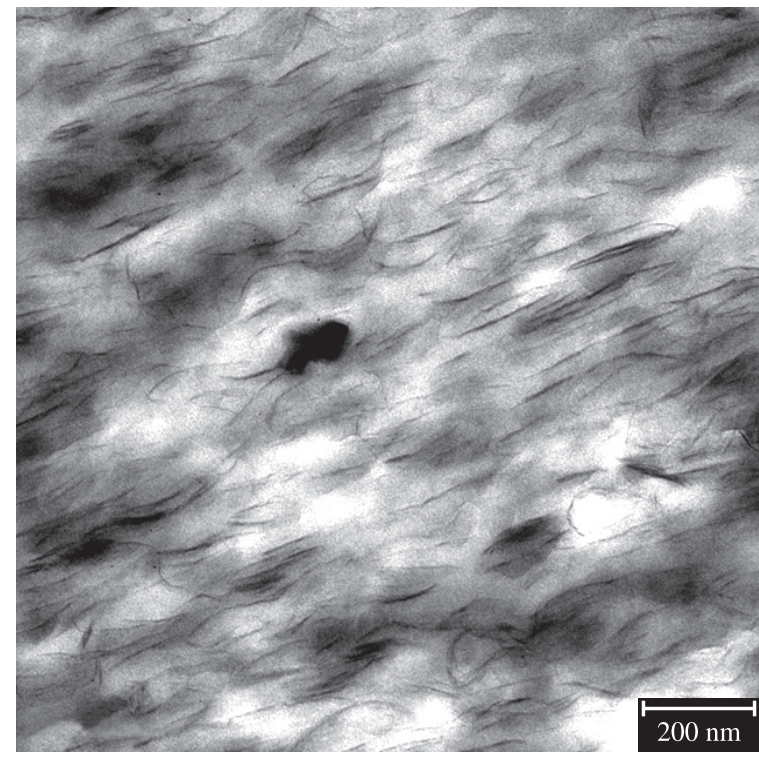

(b)

Figura 1. Micrografias de microscopia eletrônica de transmissão dos nanocompósitos: a) PVC/OMMT1; e B) PVC/OMMT5. 
da argila. Os tamanhos desses aglomerados estão na ordem de $100 \mathrm{~nm}$.

Os ensaios de análises termo-dinâmico-mecânica das amostras de composto de PVC e nanocompósitos de PVC estão demonstradas nas Figuras 2.

Como pode ser observado na Figura 2 entre a faixa de temperatura de -80 e $-45{ }^{\circ} \mathrm{C}$, ou seja, abaixo da temperatura de transição vítrea do PVC, o módulo de armazenamento do PVC/OMMT2 é discretamente menor que os outros nanocompósitos, sendo mais semelhante ao composto de PVC. Nesta região as cadeias moleculares estão estáticas, restritas a movimentações moleculares, onde a energia térmica fornecida não é suficiente para proporcionar os movimentos das cadeias moleculares.

Com o aumento da temperatura, o PVC/OMMT1 tende a uma maior sensibilidade à componente viscosa, entrando na região de transição vítrea em temperaturas menores quando comparado às outras amostras e ao composto de PVC. Nesta região o módulo tem uma queda de três ordens de grandeza, onde a energia térmica que está sendo fornecida possibilita a mobilidade das cadeias moleculares, também podendo ser considerado que a região da transição vítrea pode ser o início da movimentação molecular.

Isso quer dizer que o PVC/OMMT1 necessitou de uma menor energia térmica para dar início à mobilidade das cadeias do nanocompósito. Nesta mesma região da transição vítrea, o composto de $\mathrm{PVC}$ demonstrou maior resistência à mobilidade das cadeias. Dentre os nanocompósitos, o PVC/OMMT5 foi o que apresentou maior resistência de mobilidade das cadeias até uma temperatura de aproximadamente $-16{ }^{\circ} \mathrm{C}$.

Depois da redução do módulo durante a transição vítrea, entra-se na região do platô elástico que permanece praticamente constante até aproximadamente $50{ }^{\circ} \mathrm{C}$ para os nanocompósitos. O comprimento do platô, assim como a viscosidade, é dependente da massa molecular entre os entrelaçamentos ou reticulações. Nesta região elástica, o composto de PVC teve os maiores valores de $\tan \delta$. Com isso, pode-se dizer que, a partir desta temperatura o composto de PVC já está com alta mobilidade das cadeias e com um

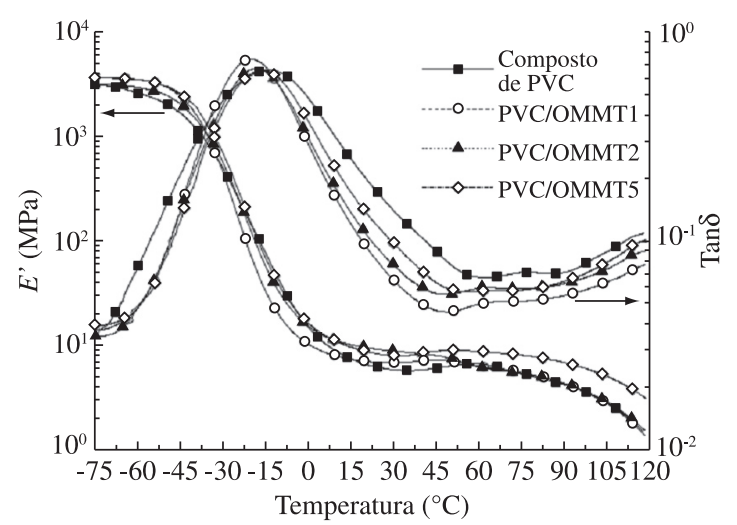

Figura 2. Módulo de armazenamento e tan $\delta$ em função da temperatura do composto de PVC e nanocompósitos. módulo de perda significativamente menor, e também uma $\tan \delta$ menor.

Em contrapartida, os nanocompósitos apresentaram maiores módulos comparado ao composto de PVC, demonstrando indícios de um enrijecimento do material. O PVC/OMMT1 foi o que apresentou menor módulo em uma faixa de temperatura entre 5 e $50{ }^{\circ} \mathrm{C}$. Nesta mesma faixa de temperatura, houve um comportamento similar para os nanocompósitos de PVC/OMMT2 e PVC/OMMT5. A partir daí, o nanocompósito de PVC/OMMT5 foi o que apresentou maior resistência ao escoamento, devido provavelmente a um aumento da componente elástica, pelo fato dos movimentos das cadeias terem sido restringidos pela formação de uma "rede física" mais compacta. Acima de $50^{\circ} \mathrm{C}$ o PVC/OMMT1 e PVC/OMMT2 tiveram comportamentos e módulos semelhantes.

Como pode ser observada, na Figura 2 a temperatura de transição vítrea dos nanocompósitos foi reduzida quando comparada ao composto de PVC em aproximadamente $4{ }^{\circ} \mathrm{C}$. Isto pode estar acontecendo devido a uma redução da interação intermolecular das camadas da OMMT na matriz do PVC e/ou a argila estar atuando como plastificante aumentando a distância entre as cadeias do PVC, resultando em uma redução na interação entre as macromoléculas. Além disso, os sais de amônio podem estar atuando como plastificantes e/ou lubrificantes, facilitando a mobilidade molecular a menores temperaturas, levando a uma redução da temperatura de transição vítrea nos nanocompósitos.

Como pode ser observado também, há um início de uma segunda transição $(T \beta)$ próximo a $40{ }^{\circ} \mathrm{C}$ para o composto de PVC, demonstrando um pico pouco intenso. Este comportamento pode estar relacionado a uma heterogeneidade na distribuição do plastificante na matriz polimérica e/ou as partículas de PVC ainda não estarem completamente gelificadas. O comportamento dinâmico-mecânico ilustrado anteriormente pode ser explicado da seguinte forma: primeiramente, a área superficial das camadas da OMMT é na faixa de 700 à $800 \mathrm{~m}^{2} \mathrm{~cm}^{-3}$, assim a imensa área interfacial entre as cadeias do polímero e da argila aumentam as limitações da conformação das cadeias do PVC nos nanocompósitos (região elástica - "rede física"). Segundo, o raio de giro das cadeias do polímero, os quais são da ordem de $10 \mathrm{~nm}$, são maiores que a distância interlamelar da OMMT $(1,8 \mathrm{~nm})$. Assim, quando as cadeias do PVC estão intercaladas entre as folhas de argila o ambiente de confinamento provavelmente restringe a mobilidade das cadeias poliméricas.

Comportamento análogo foi observado por Xu et al. ${ }^{[11]} \mathrm{e}$ Wan et al. ${ }^{[12]}$ onde se verificou que com a presença da OMMT restringiu a mobilidade das cadeias de PVC, mas a baixas temperaturas. Por outro lado, Peprnicek et al. ${ }^{[13]}$, observaram para diferentes tipos de nanocompósitos um maior efeito acima da $T g$. Diferenças significativas puderam ser relatadas a maiores temperaturas, os quais podem estar associados a diferentes tipos e níveis de interações entre a argila e a matriz polimérica. 
A Tabela 1 demonstra um resumo dos valores do módulo de armazenamento em diferentes temperaturas e a temperatura de transição vítrea para o composto de PVC e os nanocompósitos em estudo.

A degradação térmica do composto de PVC e os nanocompósitos foram caracterizados por TGA sob atmosfera de nitrogênio. Assim, podem ser visualizados na Figura 3a os termogramas de TGA para o composto de PVC, OMMT e nanocompósitos (e as respectivas primeiras derivadas, Figura $3 b$ ) em função das quantidades de argila.

Segundo Xie et al. ${ }^{[14]}$ esta degradação pode ser dividida em quatro principais estágios. $\mathrm{O}$ primeiro estágio relatado se refere à perda de umidade em temperaturas da ordem de $100{ }^{\circ} \mathrm{C}$. Os modificadores orgânicos apresentam degradação na faixa de 200 a $430{ }^{\circ} \mathrm{C}$. O estágio entre 550 a $660{ }^{\circ} \mathrm{C}$ é proveniente da desidroxilação do alumino-silicato e a evolução dos produtos associados aos resíduos carbonáceos ocorre entre 700 a $1000{ }^{\circ} \mathrm{C}$.

Para a argila em estudo, foram observados estes mesmos estágios de decomposição, com exceção da perda de água, pois a argila foi seca em estufa a vácuo antes das análises. Observaram-se, para todos os sistemas em estudo, dois estágios de degradação com comportamentos semelhantes. O primeiro estágio geralmente é atribuído à eliminação de $\mathrm{HCl}$ da cadeia do PVC, seguido pela formação de seqüências de polienos conjugados, enquanto que no segundo estágio corresponde à quebra dessas seqüências e conseqüentemente da cadeia principal.

$\mathrm{Na}$ Figura 3a, a faixa da primeira perda de massa (eliminação de $\mathrm{HCl}$ e DOP) parece sofrer pouca influência na adição de argila. Um aumento da estabilidade térmica era esperado com o aumento da concentração de OMMT. A argila pode melhorar as propriedades de barreira ao calor e transporte de massa, aumentando a estabilidade térmica do sistema e diminuindo a velocidade de degradação do polímero, como sugerido por Advani ${ }^{[15]}$.

Porém, alguns cátions metálicos presentes entre as camadas da OMMT podem catalisar a reação de desidrocloração das cadeias do $\mathrm{PVC}^{[16]}$. Além disso, os cátions orgânicos do modificador alquil amônio podem atuar como ácidos de Lewis e acelerar a liberação dos íons cloreto, removendo-os da cadeia do PVC, através da absorção na forma de um sal hidroclorídrico de amina orgânica. Este sal facilmente libera o $\mathrm{HCl}$ em altas temperaturas e induz o PVC a degradar mais rapidamente.

Tabela 1. Módulo de armazenamento e $T g$ das amostras de composto de PVC e nanocompósitos.

\begin{tabular}{ccccc}
\hline PVC/OMMT & \multicolumn{3}{c}{$E^{\prime}(\mathbf{M P a})$} & $\operatorname{Tg}\left({ }^{\circ} \mathbf{C}\right)$ \\
\cline { 2 - 4 } & $\mathbf{- 8 0}{ }^{\circ} \mathbf{C}$ & $\mathbf{2 5}^{\circ} \mathbf{C}$ & $\mathbf{1 0 0}^{\circ} \mathbf{C}$ & \\
\hline $100 / 0$ & 3.306 & 6 & 3 & $-14,4$ \\
$99 / 1$ & 3.662 & 7 & 3 & $-20,1$ \\
$98 / 2$ & 2.915 & 9 & 3 & $-18,1$ \\
$95 / 5$ & 3.570 & 8 & 6 & $-16,2$ \\
\hline
\end{tabular}

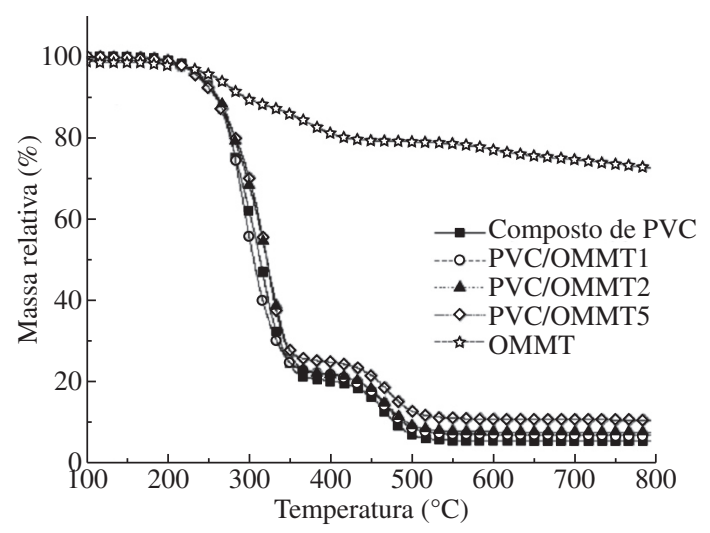

(a)

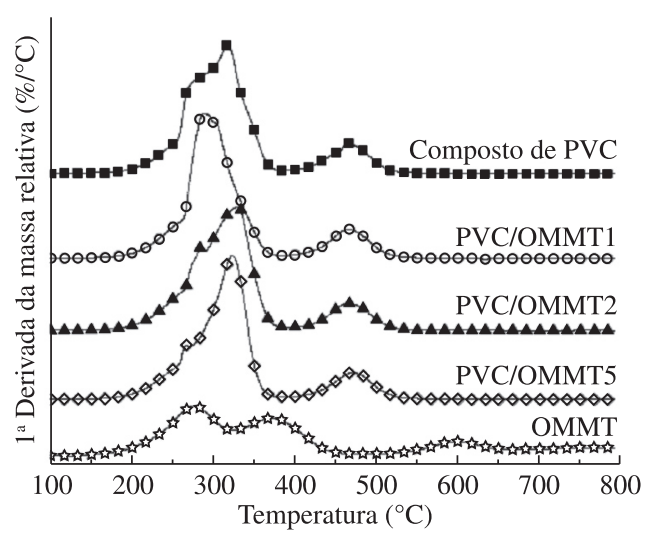

(b)

Figura 3. a) Termograma; e b) derivada de TGA para o composto de PVC, OMMT e nanocompósitos.

Nos sistemas em estudo, pode estar ocorrendo uma competição entre os dois efeitos, ou seja, entre a estabilização por aumento da barreira aos transportes de calor e massa e a catalisação da reação de degradação pela presença de ácidos de Lewis. As nanopartículas podem inibir ou melhorar a degradação da matriz polimérica, pois quanto maior o grau de intercalação das cadeias poliméricas entre as camadas da argila maior será sua razão de aspecto e a ira aumentar a estabilidade térmica do polímero. Além disso, a argila possui a capacidade de absorver moléculas pequenas (oligômeros), como por exemplo, plastificantes em sua superfície, tornando a matriz mais susceptível a degradação. No segundo estágio de degradação, o PVC/OMMT5 teve, como esperado, a menor perda de massa em relação aos outros nanocompósitos e ao composto de PVC. Isto se deve a maior quantidade de argila presente. Entretanto, pouca influência na estabilidade térmica deste estágio de decomposição foi detectada.

A Tabela 2 demonstra os valores das temperaturas iniciais dos dois estágios de degradação do composto de PVC e dos nanocompósitos. 
Tabela 2. Temperaturas iniciais de degradação do composto de PVC e nanocompósitos.

\begin{tabular}{ccc}
\hline PVC/OMMT & \multicolumn{2}{c}{ Temperatura $\left({ }^{\circ} \mathbf{C}\right)$} \\
\cline { 2 - 3 } & $T_{\text {onset1 }}$ & $T_{\text {onset2 }}$ \\
\hline $100 / 0$ & 252 & 331 \\
$99 / 1$ & 261 & 319 \\
$98 / 2$ & 252 & 341 \\
$95 / 5$ & 253 & 336 \\
\hline
\end{tabular}

\section{Conclusões}

Este trabalho procurou avaliar o efeito das concentrações de OMMT na morfologia, propriedades termo-mecânicas e termo-dinâmico-mecânicas dos nanocompósitos de PVC. Os nanocompósitos estudados demonstraram uma morfologia com presença de aglomerados quando aumentado a concentração de OMMT. Em contrapartida, obteve-se maior grau de intercalação e esfoliação aumentado a concentração da argila. Nas propriedades termo-dinâmico-mecânica, os nanocompósitos tiveram um comportamento pouco diferenciado em relação ao composto de PVC e entre os mesmos, em temperaturas abaixo da $\mathrm{Tg}$. Apresentaram maiores valores de módulo elástico para 5\% de argila e, aumentando a temperatura o nanocompósito com $1 \%$ teve menor resistência a componente viscosa entrando antes na região de transição vítrea. Em maiores temperaturas, o PVC/OMMT5 demonstrou maior resistência ao escoamento, em função possivelmente da argila ter restringido a mobilidade das cadeias. As análises termogravimétricas não demonstraram um aumento na estabilidade térmica dos nanocompósitos com a concentração de argila.

\section{Agradecimentos}

Os autores agradecem a CAPES e ao CNPQ pelo apoio financeiro, Grendene S/A (RS/Brasil), Beplast LTDA (RS/Brasil) e ao Instituto Federal Sul Rio-Grandence (IFSul) pelo fornecimento dos materiais, equipamentos e outros suportes técnicos.

\section{Referências Bibliográfica}

1. Souza, M. I.; Pessan, L. A. \& Rodolfo Júnior, A. Polímeros - Cienc Tecnol., 16, p.257 (2006).
2. Rodolfo Júnior, A. \& Mei, L. H. I. - Polímeros - Cienc Tecnol., 17, p.263 (2007).

3. Rodolfo Júnior, A.; Nunes, L. R. \& Ormanji, W. “Tecnologia do PVC”, Braskem, São Paulo (2006).

4. Mingliang, G. - J. Elastom. Plast., 40, p.223 (2008).

5. Sinha, R. S. \& Okamoto, M. - Prog. Polym. Sci., 28, p.1539 (2003).

6. Calcagno, C. I. W. - "Estudo da Morfologia, do Comportamento de Cristalização e das Propriedades Mecânicas de Nanocompósitos de PET e PP/PET com Montmorilonita", Tese de Doutorado, Universidade Federal do Rio Grande do Sul, Brasil (2007).

7. Gilman, J. W. - Appl. Clay. Sci., 15, p.31 (1999).

8. Lepoittevin, B.; Pantoustier, N.; Devalckenaere, M.; Alexandre, M.; Calberg, C.; Jérôme, R.; Henrist, C.; Rulmont, A. \& Dubois, P. - Polymer., 44, p.2033 (2003).

9. Yalcin, B. \& Cakmak, M. - Polymer., 45, p.6623 (2004).

10. Yurudu, C.; Isci, S.; Unlu, C.; Atici, O.; Ece, O. I. \& Gungor, N. - J. Appl Polym. Sci., 102, p.2315 (2006).

11. Xu, W. B.; Zhou, Z. F.; Ge, M. L. \& Pan W. P. - J. Therm. Anal. Calorim., 78, p.91 (2004).

12. Wan, C.; Qiao, X.; Zhang, Y. \& Zhang, Y. - Polym. Test., 22, p.453 (2003).

13. Peprnicek, T.; Duchet, J.; Kovarova, L.; Malac, J.; Gerard, J.F. \& Simonik, J. - Polym. Degrad. Stabil., 91, p.1855 (2006).

14. Xie, W.; Gao, Z.; Pan, W.P.; Hunter, D.; Singh, A. \& Vaia, R. - Chem. Mater., 13, p.2979 (2001).

15. Advani, S. G. - "Processing and Properties of Nanocomposites", World Scientific Publishing, Danvers (2007).

16. Leszczynska, A.; Njuguna, J.; Pielichowski, K. \& Banerjee, J. R. - Thermochim. Acta., 75, p.453 (2007).

Enviado: $31 / 10 / 09$

Reenviado: $28 / 11 / 09$

Aceito: 08/12/09

DOI: $10.1590 / \mathrm{S} 0104-14282010005000012$ 